\title{
Octahedral Transforms for 3D Image Processing
}

\author{
Reiner Lenz*, Pedro Latorre Carmona, Member, IEEE \\ EDICS: SMR-SMD Statistical-Model Based Methods \\ System and image-prior modeling;
}

\begin{abstract}
The octahedral group is one of the finite subgroups of the rotation group in three-dimensional Euclidean space and a symmetry group of the cubic grid. Compression and filtering of three-dimensional volumes are given as application examples of its representation theory. We give an overview over the finite subgroups of the three-dimensional rotation group and their classification. We summarize properties of the octahedral group and basic results from its representation theory. Widesense stationary processes are processes with group theoretical symmetries whose principal components are closely related to the representation theory of their symmetry group. Linear filter systems are defined as projection operators and symmetry-based filter systems are generalizations of the Fourier transforms. The algorithms are implemented in Maple/Matlab functions and worksheets. In the experimental part we use two publicly available MRI volumes. It is shown that the assumption of widesense stationarity is realistic and the true principal components of the correlation matrix are very well approximated by the group theoretically predicted structure. We illustrate the nature of the different types of filter systems, their invariance and transformation properties. Finally we show how thresholding in the transform domain can be used in three-dimensional signal processing.
\end{abstract}

\section{INTRODUCTION}

A LL digital image processing methods ultimately operate on discrete structures but many processing methods are based on continuous theories. This requires that the results obtained have to be adapted to the discrete case. Very often this step is essentially ignored using some ad-hoc implementations. As an example consider edge detection. Many approaches model the gray value distribution in a window as a function on a square or a disk. Edge detection is then defined as the problem to measure how similar the actual distribution is to a rotated version of an ideal function that describes an edge. But for digital images we only have values on the sampling points. Assuming the validity of the sampling theorem one can take into account the effects of sampling but then one needs extra information such as the bandwidth of the signal and smoothness conditions (see [1] for examples on the application of continuous groups in engineering).

In this paper we use an approach based on the application of finite groups (see [2], [3], [4], [5] for other applications

Corresponding author: R. Lenz is with the Department of Science and Technology, Linköping University, Norrköping, SE-601 74, Sweden, Tel: +4611-363278; Fax: +46-11-36 32 70, Email: reile@itn.liu.se

P. Latorre is with the Departamento de Lenguajes y Sistemas Informaticos, Universidad Jaume I, Campus del Riu Sec sn, 12071, Castellon de la Plana, Spain, Tel. +34 96438 7028; Email: latorre@1si.uji.es of finite groups in image and signal processing). We assume that the measurements are given on a geometrical structure with a group-theoretically defined regularity. Based on this regularity, and only on this regularity, we will then develop our image processing tools and show how they perform for real data. This is the approach used in [6], [7] to construct twodimensional filter methods. One of the main contributions of this paper is the description of the corresponding tools for the three-dimensional case where the discrete data is defined on a cubic grid. We implemented the mathematical framework in a combination of Maple and Matlab programs. Maple is used for generating the abstract description of the group involved. This description is then translated to a matrix-vector based framework which is the basis of a Matlab toolbox.

We will show that the theory predicts statistical properties that are valid for typical MRI-volumes and we will also demonstrate that the group theoretical theory provides tools that are useful in the construction of filter systems for threedimensional image processing.

The theory and, to a large extend, also the code can be directly adapted to other similar cases, for example to problems involving the icosahedral group.

\section{Finite Subgroups of SO (3)}

$\mathbf{M}$ OST digital images used today are captured by cameras built around a sensor with a square-based tiling of the sensor array. Even those using different sensor geometries are usually converted to digital images where every pixel represents a square. Such square-based tilings have a high degree of regularity: each of the squares can be rotated by 90,180 and 270 degrees and it can be mirrored along the diagonals of the square. These operations form the dihedral group $D(4)$ consisting of eight elements describing all similarity operations of a square. Based on this group a class of images processing methods were developed in [6], [7]. These methods are essentially the transforms for the square geometry corresponding to Fourier and Fast Fourier Transforms for signals defined on a line or a circle. The starting point for the theory presented here is the observation that the rotations mapping the square grid into itself are the $0,90,180$ and 270 degrees rotations and that these form a four-element subgroup of the continuous group SO (2) of 2D-rotations.

Generalizing this approach to 3D we will first characterize the finite subgroups of the 3D rotation group $\mathrm{SO}(3)$. We will then describe the detailed structure of one of these groups, 
the Octahedral group $\mathrm{O}$ and we will explain its relation to the cubic sampling pattern in 3D space.

We now introduce some notations and basic facts about groups in general and the three-dimensional rotation group in particular. Rotations can be described by those $n \times n$ matrices $\boldsymbol{R}$ with determinant equal to one that satisfy the equation $\boldsymbol{R} \boldsymbol{R}^{\prime}=\boldsymbol{I}$ where $\boldsymbol{R}^{\prime}$ denotes transposition and $\boldsymbol{I}$ is the identity matrix. These matrices form the group $\mathrm{SO}(n)$. Here we are only interested in the case SO (3). Rotations preserve the Euclidean length of vectors and therefore the unit sphere $\mathcal{S}=\{\boldsymbol{x}:\|\boldsymbol{x}\|=1\}$ is preserved under rotations.

The rotations operate on the sphere and we define the transformation group as the pair $(\operatorname{SO}(3), \mathcal{S})$ with the group action $(\boldsymbol{R}, \boldsymbol{x}) \mapsto \boldsymbol{R} \boldsymbol{x}$. If $G$ is a subgroup of SO (3) and $\boldsymbol{x} \in \mathcal{S}$ is a unit vector then we define the orbit of $\boldsymbol{x}$ under $G$ as: $G \boldsymbol{x}=\{\boldsymbol{R} \boldsymbol{x}: \boldsymbol{R} \in G\}$.

From basic geometry we know that each three-dimensional rotation (different from the identity) has a rotation axis and that there are exactly two points on the unit sphere that are left invariant under a given rotation. We consider now a subgroup $G$ of SO (3) that has finitely many elements and we denote the number of group elements by $n$. For an element $\boldsymbol{x} \in \mathcal{S}$ and a group $G$ we define its stabilizer $G_{x}$ as the group of all elements in $G$ leaving $\boldsymbol{x}$ fixed: $G_{x}=\{\boldsymbol{R} \in G: \boldsymbol{R} \boldsymbol{x}=\boldsymbol{x}\}$.

The finite subgroups of SO (3) are characterized with the help of their orbits and stabilizers. The complete derivation can be found in [8], see also [9]. We consider a finite subgroup $G$ of SO (3) with $n$ elements. We define $P$ as the set of points on the sphere that are invariant under at least one element in $G: P=\{\boldsymbol{x} \in \mathcal{S}: \exists \boldsymbol{R} \in G: \boldsymbol{R} \boldsymbol{x}=\boldsymbol{x}\}$ and the set $G P=$ $\{(\boldsymbol{R}, \boldsymbol{x}): \boldsymbol{R} \in G, \boldsymbol{R} \neq \boldsymbol{I}, \boldsymbol{x} \in P\}$. Every rotation in $G P$ has two fixed points and $G P$ consists of $2(n-1)$ point pairs. This construction contains whole orbits and if we denote the number of orbits in $P$ by $r$ and the number of group elements in the stabilizer of a point on orbit number $i$ by $n_{i}$ then we obtain the following equation $2(n-1)=\sum_{i=1}^{r}\left(n-\frac{n}{n_{i}}\right)$. This relation between $n, r$ and the $n_{i}$ 's implies as only solutions: $r=2$ and $r=3$. For $r=2$ the group is a group around one fixed axis. For $r=3$ one can assume $n_{1} \leq n_{2} \leq n_{3}$ and finds $n_{1}=2, n_{2}=3$ and $1 / n_{3}=1 / 6+2 / n=(1+(12 / n)) / 6$ and therefore $n_{3}<6$. The three possible combinations $\left(n_{1}, n_{2}, n_{3}\right)$ are $(2,3,3),(2,3,4),(2,3,5)$ with 12,24 and 60 elements. Here we consider the case $(2,3,4)$ defining the Octahedral group $\mathrm{O}$, for the other cases (especially the icosahedral group of type $(2,3,5)$ with 60 elements) we refer the reader to [9], [8] and also [10].

\section{BASIC FACTS ABOUT THE OCTAHEDRAL GROUP}

W E now collect a few facts about $\mathrm{O}$ that will be needed later (for details see for example [11], [9], [12] or [8]).

We say that a group is generated by some group elements if all group elements can be written as a combination of these elements and their inverses. Such elements are the generators of the group. The abstract group $\mathrm{O}$ is defined by two generators $A, D$ and four defining relations. In Maple code:

OGroup := grelgroup $(\{\mathrm{A}, \mathrm{D}\}$,

$$
\begin{aligned}
& \{[D, D, D],[A, A, A, A], \\
& {[A, D, D, A, 1 / D],} \\
& [A, D, A, 1 / D, 1 / A, 1 / A, 1 / D]\}) ;
\end{aligned}
$$

which means that the two group elements $A, D$ satisfy the equations

$$
\begin{aligned}
D D D & =A A A A=A D D A D^{-1}= \\
& =A D A D^{-1} A^{-1} A^{-1} D^{-1}=I
\end{aligned}
$$

where $I$ represents the identity element of the group. The Maple code generates an abstract internal description of this group.

In the case where the abstract group is represented by $3 \mathrm{D}$ rotation matrices, the equation $D D D=I$ means that the corresponding rotation matrix $\boldsymbol{R}_{1}$ must be a 120 degree rotation around some axis. The second generator equation implies that $A$ corresponds to a 90 degree rotation. A simple computation shows that the following two rotation matrices satisfy these equations: the rotation $\boldsymbol{R}_{1}$ around the diagonal and $\boldsymbol{R}_{2}$, a rotation around the $z$-axis.

$$
\begin{aligned}
D \rightarrow \boldsymbol{R}_{1} & =\left(\begin{array}{ccc}
0 & 0 & 1 \\
1 & 0 & 0 \\
0 & 1 & 0
\end{array}\right) \\
A \rightarrow \boldsymbol{R}_{2} & =\left(\begin{array}{ccc}
0 & 1 & 0 \\
-1 & 0 & 0 \\
0 & 0 & 1
\end{array}\right)
\end{aligned}
$$

Next consider a finite collection $W$ of points $\boldsymbol{x}$ in space and assume that it is invariant under operations of $\mathrm{O}: \boldsymbol{R} \boldsymbol{x} \in$ $W, \forall \boldsymbol{R} \in \mathrm{O}$ and $\forall x \in W$. This is a special example of a group $G$ operating on a set $S$, defined as the existence of a mapping $(G, S) \rightarrow S ;(g, s) \mapsto g s$ such that $(g h) s=$ $g(h(s)), \forall g, h \in G, \forall s \in S$.

In the following we make use of the smallest possible set $W=G s_{0}=\left\{g s_{0}, g \in G\right\}$, the orbit of a single element. For the Octahedral group $\mathrm{O}$ consider as an example a corner point $\boldsymbol{x}_{0}$ of the cube with coordinate vector $\boldsymbol{x}_{0}=(1,1,1)$. We see immediately that both rotations $\boldsymbol{R}_{1}, \boldsymbol{R}_{2}$ defined in Eq. (1) map this corner point to another corner point of the cube. Applying all combinations of $\boldsymbol{R}_{1}, \boldsymbol{R}_{2}$ we can reach all corner points of the cube. The corner points of the cube form therefore an orbit consisting of eight points.

To get an overview over all orbits of $\mathrm{O}$ on the cubic grid we consider a general point $\boldsymbol{x}_{0}$ with coordinate vector $\boldsymbol{x}_{0}=(\xi, \eta, \zeta)$. We see that $\boldsymbol{R}_{1}$ defines a shift of the components of the coordinate vector and that $\boldsymbol{R}_{2}$ permutes them and changes the sign of one of the components. Using combinations of the shifts and the sign change it is easy to see that we can first generate eight variants of the coordinate vector by switching signs of the coordinate values. Then we can identify a unique element in the coordinate vector and shift it with the help of $\boldsymbol{R}_{1}$ to the first position in the coordinate vector. With this we have shown that on each orbit we can find an element with coordinate vector $(\xi, \eta, \zeta)$ such that $0 \leq \xi \leq \eta, \zeta$ where the last inequality means that both $\eta$ and $\zeta$ are greater than or equal to $\xi$ but no ordering between $\eta, \zeta$ is given. Vectors $(1,2,3)$ and $(1,3,2)$ are thus 
two such coordinate vectors and they define different orbits. An easy calculation shows however that two vectors $(0, \zeta, \eta)$ and $(0, \eta, \zeta)$ lie on the same orbit. Arbitrary O-invariant subsets of the space can thus be characterized by sets

$$
\left\{\left(\xi_{k}, \eta_{k}, \zeta_{k}\right): 0 \leq \xi_{k} \leq \eta_{k}, \zeta_{k}, k=1, \ldots K\right\}
$$

For $(0, \eta, \zeta)$ we require $\eta<\zeta$.

We have the following theorem:

Theorem $1 \mathrm{O}$ has 24 elements and can be described by the two generators $\boldsymbol{R}_{1}, \boldsymbol{R}_{2}$ and four equations.

Elements in $\mathrm{O}$ map points on the cubic grid to other points on the cubic grid. It has five different types of orbits: (i) The single point orbit $\mathcal{O}_{o}$ consisting of the origin. (ii) The axis orbit $\mathcal{O}_{a}$ of the six points on the coordinate axes. They are the centers of the faces of the cube. (iii) The corner orbit $\mathcal{O}_{c}$ consisting of the eight corner points of a centered cube. (iv) The vertex orbit $\mathcal{O}_{v}$ with the twelve points on the middle of the vertices. Finally (v) The general orbit $\mathcal{O}_{g}$ with 24 points.

Consider again a finite collection $W$ of $N$ points in space that is invariant under all operations of the octahedral group $\mathrm{O}$. The scalar valued functions defined on $W$ form an $N$-dimensional vector space. A function $f$ defined on $W$ can be described by an $N$-dimensional vector that we will also denote by $f$. These functions form the vector space $\mathbb{R}^{N}$. The rotations $\boldsymbol{R}$ in the group $\mathrm{O}$ operate on $W$ and therefore also on the functions on $W$. We illustrate this by constructing the matrix acting on a six-point axis orbit $\mathcal{O}_{a}$. We denote the six points on the $\mathrm{x}-, \mathrm{y}-$ and z-axis as $\boldsymbol{x},-\boldsymbol{x}, \boldsymbol{y},-\boldsymbol{y}, \boldsymbol{z}$ and $-\boldsymbol{z}$ and order them as $\boldsymbol{x},-\boldsymbol{y},-\boldsymbol{x}, \boldsymbol{y}, \boldsymbol{z}$ and $-\boldsymbol{z}$. The ordering of these points is arbitrary and we only choose it to get a simple matrix description later. In this order we map the six points to the six canonical basis vectors $e_{1}, \ldots, e_{6}$ in a six-dimensional vector space. Thus: $\boldsymbol{x} \mapsto \boldsymbol{e}_{\mathbf{1}},-\boldsymbol{y} \mapsto \boldsymbol{e}_{\mathbf{2}}, \ldots, \boldsymbol{z} \mapsto \boldsymbol{e}_{\mathbf{6}}$. A simple calculation shows now that if we apply the rotation $\boldsymbol{R}_{2}$ to the coordinate vectors then the matrix describing the transformation of the values on the orbit is (in this ordering) given by

$$
\left(\begin{array}{llllll}
0 & 0 & 0 & 1 & 0 & 0 \\
1 & 0 & 0 & 0 & 0 & 0 \\
0 & 1 & 0 & 0 & 0 & 0 \\
0 & 0 & 1 & 0 & 0 & 0 \\
0 & 0 & 0 & 0 & 1 & 0 \\
0 & 0 & 0 & 0 & 0 & 1
\end{array}\right)
$$

In this way we see that for every function $f$, defined on an invariant set with $N$ - points, and every rotation $\boldsymbol{R}$ we get a transformed function $f^{\boldsymbol{R}}: f^{\boldsymbol{R}}(\boldsymbol{x})=f\left(\boldsymbol{R}^{-1} \boldsymbol{x}\right)$. Every rotation $\boldsymbol{R}$ defines therefore an $N \times N$ matrix $\boldsymbol{T}(\boldsymbol{R})$ such that $f^{\boldsymbol{R}}=\boldsymbol{T}(\boldsymbol{R}) f$. We formalize this in the definition of a representation of a group:

Definition 1 A matrix representation of a group is a mapping $\boldsymbol{T}: g \mapsto \boldsymbol{T}(g)$ with

$$
\boldsymbol{T}(g h)=\boldsymbol{T}(g) \boldsymbol{T}(h) \forall g, h \in G
$$

where the $\boldsymbol{T}(g)$ are invertible matrices. If the matrices $\boldsymbol{T}(g)$ are of size $N \times N$ then we say that the degree of the representation is $N$.
From these definitions it can be seen why we used the inverse in $f^{\boldsymbol{R}}(\boldsymbol{x})=f\left(\boldsymbol{R}^{-1} \boldsymbol{x}\right)$. We have for arbitrary elements $Q_{1}, Q_{2}$ :

$$
\begin{aligned}
& \left(\boldsymbol{T}\left(\boldsymbol{Q}_{\mathbf{1}} \boldsymbol{Q}_{\mathbf{2}}\right) f\right)(\boldsymbol{x})=f\left(\left(\boldsymbol{Q}_{\mathbf{1}} \boldsymbol{Q}_{\mathbf{2}}\right)^{-1} \boldsymbol{x}\right)=f\left(\boldsymbol{Q}_{\mathbf{2}}{ }^{-1} \boldsymbol{Q}_{\mathbf{1}}{ }^{-1} \boldsymbol{x}\right) \\
& \quad=\left(\boldsymbol{T}\left(\boldsymbol{Q}_{\mathbf{2}}\right) f\right)\left(\boldsymbol{Q}_{\mathbf{1}}{ }^{-1} \boldsymbol{x}\right)=\left(\boldsymbol{T}\left(\boldsymbol{Q}_{\mathbf{1}}\right)\left(\boldsymbol{T}\left(\boldsymbol{Q}_{\mathbf{2}}\right) f\right)\right)(\boldsymbol{x}) \\
& \quad=\left(\left(\boldsymbol{T}\left(\boldsymbol{Q}_{\mathbf{1}}\right) \boldsymbol{T}\left(\boldsymbol{Q}_{\mathbf{2}}\right)\right) f\right)(\boldsymbol{x})
\end{aligned}
$$

For a given orbit we can now construct the representation matrices $\boldsymbol{T}\left(\boldsymbol{R}_{1}\right), \boldsymbol{T}\left(\boldsymbol{R}_{2}\right)$ and since all rotations $\boldsymbol{R}$ are products of $\boldsymbol{R}_{1}, \boldsymbol{R}_{2}$ and their inverses and since $\boldsymbol{T}\left(\boldsymbol{R}_{1}\right) \boldsymbol{T}\left(\boldsymbol{R}_{2}\right)=$ $\boldsymbol{T}\left(\boldsymbol{R}_{1} \boldsymbol{R}_{2}\right)$ we see that we can generate all matrices $\boldsymbol{T}(\boldsymbol{R})$ as products of $\boldsymbol{T}\left(\boldsymbol{R}_{1}\right), \boldsymbol{T}\left(\boldsymbol{R}_{2}\right)$ or their inverses. Often the representation matrices are permutation matrices and their inverses are given by the transpose. Using this construction we see that we can create for each orbit a representation of the group $\mathrm{O}$ by constructing the corresponding permutation matrices for the generating rotations $\boldsymbol{R}_{1}, \boldsymbol{R}_{2}$. For arbitrary sets $W$ of points we partition $W$ first into disjunct subsets consisting of orbits and then we construct the representations of each of the orbits separately. The representation on the whole set is the direct product of these orbit representations. In practice we have to construct 24 matrices, one for each element in $\mathrm{O}$ and for sets $W$ with many grid points these matrices can be quite large (an $8 \times 8 \times 8$ cube requires matrices $\boldsymbol{T}(\boldsymbol{R})$ of size $512^{2}$ ). We will therefore compute them automatically in a series of Maple and Matlab programs. We will consider two problems as illustrations, approximations of Principal Component Analysis (PCA) and filtering.

\section{Wide-Sense Stationary Processes and Partial PRINCIPAL COMPONENTS}

$\mathbf{W}$ E consider again a set $W$ of points invariant under $\mathrm{O}$ and the functions/vectors $f$ defined on $W$. We assume that the $f$ are outcomes of a stochastic process and sometimes we write $f_{\omega}$ where $\omega$ is the stochastic variable. By $\boldsymbol{C}$ we denote the matrix of second order moments. We call this the correlation matrix of the process and we have

$$
\boldsymbol{C}=\mathrm{E}\left(f_{\omega} f_{\omega}^{\prime}\right)
$$

where $\mathrm{E}(\mathrm{)}$ denotes the expectation operator. If we apply a transformation $\boldsymbol{T}(\boldsymbol{R})$ to all the vectors first we get a new matrix $C^{\boldsymbol{R}}=\boldsymbol{T}(\boldsymbol{R}) \boldsymbol{C T}(\boldsymbol{R})^{\prime}$ and if all the rotated versions of $f$ occur with the same probability in the original process then the application of the transformations $\boldsymbol{T}(\boldsymbol{R}) f$ results only in a reordering of the vectors and we have

$$
\boldsymbol{C}=\boldsymbol{C}^{\boldsymbol{R}}=\boldsymbol{T}(\boldsymbol{R}) \boldsymbol{C T}(\boldsymbol{R})^{\prime} \forall \boldsymbol{R} \in \mathrm{O}
$$

A stochastic process that satisfies the conditions in Eq.( 4) is an $\mathrm{O}$-wide-sense stationary process. Correlation matrices of $\mathrm{O}$-wide-sense stationary processes are thus the solutions $C$ of these 24 matrix equations (see also [13], [14]).

Before we can characterize these correlation matrices we need a number of characteristics of the transformations $\boldsymbol{T}(\boldsymbol{R})$. The $\boldsymbol{T}(\boldsymbol{R})$ are matrices of size $N \times N$ and as such they define linear mappings $\boldsymbol{T}(\boldsymbol{R}): V \rightarrow V$ where $V$ is an $N$-dimensional vector space. If we change the basis in this vector space $V$ with the help of a matrix $B$ then the new 
matrices $\boldsymbol{B} \boldsymbol{T}(\boldsymbol{R}) \boldsymbol{B}^{-1}$ describe the same linear mappings of the vector space but in this new basis. In the following we consider mainly orthonormal matrices $\boldsymbol{B}$ for which this is equivalent to $\boldsymbol{B} \boldsymbol{T}(\boldsymbol{R}) \boldsymbol{B}^{\prime}$. We call $\boldsymbol{T}$ a reducible representation if we can find a matrix $\boldsymbol{B}$ such that

$\boldsymbol{B} \boldsymbol{T}(\boldsymbol{R}) \boldsymbol{B}^{-1}=\left(\begin{array}{cc}\boldsymbol{T}_{1}(\boldsymbol{R}) & 0 \\ 0 & \boldsymbol{T}_{2}(\boldsymbol{R})\end{array}\right)=\operatorname{diag}\left(\boldsymbol{T}_{1}(\boldsymbol{R}), \boldsymbol{T}_{2}(\boldsymbol{R})\right)$

where $\boldsymbol{T}_{k}(\boldsymbol{R})$ is of size $N_{k}$ with $0<N_{1}, N_{2}$, and $N_{1}+N_{2}=$ $N$. This means we can split the original vector space into two subspaces such that the original representation defines two new representations of lower dimensions, one on each of these subspaces. A representation that is not reducible is called irreducible. A key result from the theory of group representations is the following theorem [11]:

Theorem 2 Assume $\boldsymbol{T}$ is a unitary irreducible representation of $\mathrm{O}$ and $\boldsymbol{C}$ is a matrix with

$$
\boldsymbol{C}=\boldsymbol{T}(\boldsymbol{R}) \boldsymbol{C T}(\boldsymbol{R})^{-1} \forall \boldsymbol{R} \in \mathrm{O}
$$

then $C$ is the zero matrix or a multiple of the identity matrix $\boldsymbol{I}$, i.e. there is a constant $\lambda$ such that $\boldsymbol{C}=\lambda \boldsymbol{I}$.

We use this to describe the structure of a matrix $C$ that satisfies $\boldsymbol{C}=\boldsymbol{T}(\boldsymbol{R}) \boldsymbol{C T}(\boldsymbol{R})^{\prime} \quad \forall \boldsymbol{R} \in \mathrm{O}$ and $\boldsymbol{T}(\boldsymbol{R})$ orthonormal. It can be shown that we can find an orthonormal matrix $B$ such that that $\boldsymbol{B} \boldsymbol{T}(\boldsymbol{R}) \boldsymbol{B}^{\prime}=\operatorname{diag}\left(\boldsymbol{T}_{1}(\boldsymbol{R}), \boldsymbol{T}_{2}(\boldsymbol{R}), \ldots, \boldsymbol{T}_{K}(\boldsymbol{R})\right)$ where all representations $\boldsymbol{T}_{k}$ are irreducible. We then find from $C=\boldsymbol{T}(\boldsymbol{R}) \boldsymbol{C T}(\boldsymbol{R})^{\prime}$ :

$$
\begin{gathered}
\boldsymbol{B C} \boldsymbol{B}^{\prime}=\boldsymbol{B} \boldsymbol{T}(\boldsymbol{R}) \boldsymbol{C} \boldsymbol{T}(\boldsymbol{R})^{\prime} \boldsymbol{B}^{\prime}=\boldsymbol{B} \boldsymbol{T}(\boldsymbol{R}) \boldsymbol{B}^{\prime} \boldsymbol{B} \boldsymbol{C} \boldsymbol{B}^{\prime} \boldsymbol{B} \boldsymbol{T}(\boldsymbol{R})^{\prime} \boldsymbol{B}^{\prime} \\
=\operatorname{diag}\left(\boldsymbol{T}_{1}(\boldsymbol{R}), \boldsymbol{T}_{2}(\boldsymbol{R}), \ldots, \boldsymbol{T}_{K}(\boldsymbol{R})\right) \\
\boldsymbol{B C}^{\prime} \cdot \operatorname{diag}\left(\boldsymbol{T}_{1}(\boldsymbol{R}), \boldsymbol{T}_{2}(\boldsymbol{R}), \ldots, \boldsymbol{T}_{K}(\boldsymbol{R})\right)^{\prime}
\end{gathered}
$$

or

$$
\begin{array}{r}
\widetilde{\boldsymbol{C}}=\operatorname{diag}\left(\boldsymbol{T}_{1}(\boldsymbol{R}), \boldsymbol{T}_{2}(\boldsymbol{R}), \ldots, \boldsymbol{T}_{K}(\boldsymbol{R})\right) \\
\widetilde{\boldsymbol{C}} \operatorname{diag}\left(\boldsymbol{T}_{1}(\boldsymbol{R}), \boldsymbol{T}_{2}(\boldsymbol{R}), \ldots, \boldsymbol{T}_{K}(\boldsymbol{R})\right)^{\prime}
\end{array}
$$

If we now split the new correlation matrix $\widetilde{\boldsymbol{C}}$ into blocks $\widetilde{\boldsymbol{C}}_{m n}$ corresponding to the sizes of the irreducible representations $\boldsymbol{T}_{k}$ then we find for these blocks the equations

$$
\widetilde{\boldsymbol{C}}_{m n} \boldsymbol{T}_{n}=\boldsymbol{T}_{m} \widetilde{\boldsymbol{C}}_{m n}
$$

For $m, n$ for which the $\boldsymbol{T}_{m}, \boldsymbol{T}_{n}$ are not equivalent we find $\widetilde{\boldsymbol{C}}_{m n}=0$ from the previous theorem.

Summarizing we see that for a wide-sense stationary process, a representation $\boldsymbol{T}$ of $\mathrm{O}$ and an invariant correlation matrix $C$ of size $N \times N$ we can construct an orthonormal matrix $B$ that splits the underlying space into subspaces of dimensions $N_{1}, \ldots, N_{K}, N_{1}+\cdots+N_{K}=N$ such that the resulting new representations $\boldsymbol{T}_{1}, \ldots, \boldsymbol{T}_{K}$ are irreducible and the transformed correlation matrix $\widetilde{C}=B C B^{\prime}$ is blockdiagonal where the structure of the blocks is only given by the group and the size $N$.

We analyze wide-sense stationary processes by computing this decomposition in two steps: in the first step the point set on which the process is defined is divided into components defined by the orbits. According to Theorem 1 this gives five types of subspaces of dimensions $1,6,8,12$ and 24 . The subdivision of the four spaces with dimension greater than one is then obtained using the following definitions and a projection theorem (see [11], [15]).

There are five irreducible representations of $\mathrm{O}$ given by $\boldsymbol{T}_{k}, k=1, \ldots, 5$. We denote the dimension of $\boldsymbol{T}_{k}$ by $n_{k}$. These dimensions are $1,1,2,3,3$. We denote the vector space on which $\boldsymbol{T}_{k}$ is defined by $W_{k}$. For a general representation $\boldsymbol{T}$ we denote the vector space on which it is defined by $V$. The decomposition of $\boldsymbol{T}$ into irreducible representations defines a decomposition of this vector space

$$
V=U_{1} \bigoplus \ldots \bigoplus U_{L}
$$

where each of the $U_{l}$ is one of the $W_{k}$. We now collect all $U_{l}$ that correspond to a given $W_{k}$ into the direct sum

$$
V_{k}=U_{k 1} \bigoplus \ldots \bigoplus U_{k M}
$$

with $U_{k m}$ equivalent to $W_{k}$. The canonical decomposition of $V$ is given by $V=V_{1} \oplus \ldots \oplus V_{5}$. The canonical decomposition of $V$ is computed with the help of characters, which we introduce now.

The trace $\operatorname{tr}(\boldsymbol{M})$ of a matrix $\boldsymbol{M}$ is the sum of its diagonal elements. For a matrix representation $\boldsymbol{T}$ of the group $\mathrm{O}$ this gives a mapping defined on the group $\boldsymbol{R} \mapsto \chi(\boldsymbol{R})=$ $\operatorname{tr}(\boldsymbol{T}(\boldsymbol{R}))$. This mapping is the character of the representation. The complex valued functions on $\mathrm{O}$ define a vector space and on this vector space we define the scalar product $\left\langle f_{1}, f_{2}\right\rangle=$ $\frac{1}{24} \sum_{\boldsymbol{R} \in \mathrm{O}} f_{1}(\boldsymbol{R}) \overline{f_{2}(\boldsymbol{R})}$ and the characters of the irreducible representations form an orthonormal system in this vector space. This gives:

Theorem 3 The Octahedral group O has five basic irreducible representations $\boldsymbol{T}_{k}, k=1, \ldots, 5$ of dimensions 1, 1,2, 3 and 3 . We denote their characters by $\chi_{k}$. For a general representation $\boldsymbol{T}$ (on the vector space $V$ ) the canonical decomposition is unique and the projection operator

$$
p_{k}=\frac{n_{k}}{24} \sum_{\boldsymbol{R} \in \mathrm{O}} \overline{\chi_{k}(\boldsymbol{R})} \boldsymbol{T}(g)
$$

defines the projection from the original space $V$ to the invariant subspace given by $V_{k}$.

\section{FILTERING}

$\mathbf{I}$ $\mathrm{N}$ the last section we described how to use the representation theory of the octahedral group $\mathrm{O}$ to block-diagonalize the correlation matrix of a wide-sense stationary process. This can be used for transform coding or an approximation of principal component analysis where the solution of one large eigenvector problem is reduced to the solution of five smaller eigenvector equations.

In this section we will now describe how the Octahedral group can be used in low-level filtering. One of the basic strategies in filtering is to use systematic variations of a given filter. Typical examples are wavelets and Gabor analysis where filters of different orientation, frequency and scale are applied. Here we will use the properties of the Octahedral group to design filter systems with similar transform properties. 
As before we use a set $W$ of $N$ points in space that is invariant under all rotations of $\mathrm{O}$. The functions defined on $W$ are described by vectors in the $N$-dimensional vector space $V$. On $V$ we have the representation $\boldsymbol{T}$ transforming functions $f$ under the rotation $\boldsymbol{R}$ as $f \mapsto \boldsymbol{T}(\boldsymbol{R}) f$.

For filter design we now consider a fixed function $f$ and its orbit $f^{\boldsymbol{R}}, \boldsymbol{R} \in \mathrm{O}$. This defines a filter system with 24 filter functions. Depending on the nature of $f$ these are, however, not necessarily all different. All functions $f^{\boldsymbol{R}}$ lie in $V$ and they span an invariant subspace $V_{f}$ of $V$. From the general theory we know that $\boldsymbol{T}$ defines a partition of $V$ into smallest $\mathrm{O}$ invariant subspaces of dimensions $1,1,2,3,3$ given by the five irreducible representations of $\mathrm{O}$. We denote the transformation matrix from the ordinary, voxel-based, coordinate system to the system given by the irreducible representations by $\boldsymbol{B}$. This is a matrix of size $N \times N$ and we can assume that it is orthonormal. Furthermore, we order the matrix $\boldsymbol{B}$ such that the transformed vector $\boldsymbol{B} f$ has the form $\boldsymbol{B} f=\left(f^{1}, f^{2}, f^{3}, f^{4}, f^{5}\right)$ where $f^{k}$ is the projection of the vector $f$ to that part of the vector space that transforms like the $k$-th irreducible representation of $\mathrm{O}$. If we denote the length of $f^{k}$ by $N_{k}$ then we have $N=N_{1}+\ldots N_{5}$. To simplify notations we denote the two matrices that represent the group generators in the representation $T$ by $A$ and $D: A=T\left(R_{1}\right), D=T\left(R_{2}\right)$ (see Equation (1)).

With these notations we can now describe the implementation of a filter system $f^{\boldsymbol{R}}$ based on the transformation properties of the octahedral group. Using this decomposition we will see how many filter functions are actually necessary to implement the filter system and we will also see that the different sub-systems have transformation properties that allow us to extract information about the underlying orientation of the pattern.

As an illustration of the construction of a group theoretical filter system we start with a given filter function $f$ that defines the orbit $f^{\boldsymbol{R}}, \boldsymbol{R} \in \mathrm{O}$. A typical example could be an edge filter in the $\mathrm{x}$-direction. We now describe one method to construct the symmetry-adapted filter system:

1) For $f$ use the transformation $\boldsymbol{B} f$ and compute $\boldsymbol{B} f=\left(f^{1}, f^{2}, f^{3}, f^{4}, f^{5}\right)$

2) Select that part $\hat{f}$ in $\boldsymbol{B} f=\left(f^{1}, f^{2}, f^{3}, f^{4}, f^{5}\right)$ that has the highest norm of these five components

3) Compute the projection on the subspace with the highest projection norm by keeping component $\hat{f}$ and replacing the rest by zero-vectors. This gives the projected vector $f^{p}$

4) Reconstruct the corresponding vector in the original space as $f_{p}=\boldsymbol{B}^{\prime} f^{p}$ (Note that an orthonormal $\boldsymbol{B}$ is used)

5) For the cases $\hat{f}=f^{1}$ and $\hat{f}=f^{2}$ use only $f_{p}=\boldsymbol{B}^{\prime} f^{p}$ since the application of transformed versions of $f_{p}$ will at most change sign of the filter result

6) For the case $\hat{f}=f^{3}$ the representation space is twodimensional and therefore $f_{p}=\boldsymbol{B}^{\prime} f^{p}$ and $\boldsymbol{D} f_{p}$ are used

7) For the case of the fourth irreducible representation $\hat{f}=$ $f^{4}$ use the three filters $f_{p}=\boldsymbol{B}^{\prime} f^{p}, \boldsymbol{D} \boldsymbol{A} f_{p}$ and $\boldsymbol{D} f_{p}$

8) For the remaining case $\hat{f}=f^{5}$ use the three filters $f_{p}=$
$\boldsymbol{B}^{\prime} f^{p}, \boldsymbol{D} f_{p}$ and $\boldsymbol{A} f_{p}$

9) The result of the construction in the last four steps is now a one-, two- or three-dimensional filter matrix $\boldsymbol{F}_{p}$

10) Finally update the filter vector $f$ by projecting it to the orthogonal complement of $\boldsymbol{F}_{p}$

11) If the resulting projected vector is not zero then repeat the construction and compute the next filter matrix $\boldsymbol{F}_{p}$

Using this projection technique we can always assume that the computed filter vectors transform like the corresponding irreducible representation. We denote such a projection-based filter system by $\boldsymbol{F}^{k}$ where $0 \leq k \leq 5$ denotes the index of the irreducible representation. We now describe how to use the computed filter vectors in further processing based on their transformation properties.

The filter systems of type $\boldsymbol{F}^{1}, \boldsymbol{F}^{2}$ consist of one filter function only. The results of filtering with the first system $\boldsymbol{F}^{1}$ are independent of orientation changes of the underlying signal. We therefore use the filter results as they are. Filter results obtained from $\boldsymbol{F}^{2}$ are also scalar-valued. Their behaviour under rotations is described with the help of cosets defined as follows: Given a subgroup $H$ of a group $G$ we define an equivalence relation on $G$ by defining $g_{1}, g_{2}$ as equivalent if there is an element $h \in H$ such that $g_{2}=g_{1} h$. The filter results for the second filter type are invariant under one subgroup of $\mathrm{O}$ and change sign for the other coset of this subgroup. Therefore we describe the filter results by their absolute values and their signs. The sign indicates which coset of rotations was underlying the actual pattern. Processing of the remaining, higher-dimensional, filter vectors is more complicated.

For the two-dimensional filter systems $\boldsymbol{F}^{3}$ the filter vectors transform under rotations in $\mathrm{O}$ as the irreducible twodimensional representation. The 24 transformation matrices come in classes of four identical matrices each. The six different classes are represented by the three rotations with rotation angles 0,120 and 240 degrees and by the same three rotations combined with a sign-change in the first coordinate. From this we derive the following coding of the two-dimensional filter result vectors: First collect the two filter results in the vector $\left(f_{1}, f_{2}\right)$ and convert them to polar coordinates $(r, \theta)$. The value of $r$ is independent of group transformations of the underlying domain and is an indicator of how good the overall fit of the value distribution and the whole filter system was. For the $\theta$ angle we first split the unit circle into six equally long (60 degrees) segments. The rotation part of the transformation can be used to move a given $\theta$-value into one of the three adjoining (120 degrees) segments. Using a possible signchange we can always achieve that the final transformation of the angle is in one of the two (60 degrees) segments. The location of the final transform within this segment is described by a number that we call the residue. The angle is therefore coded into an integer label $0, \ldots 5$ and a residue value $\rho$. The coding of $\theta$ is described in the following Matlab code:

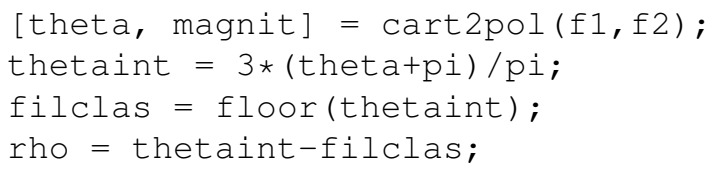

The filter system derived from the fourth irreducible repre- 


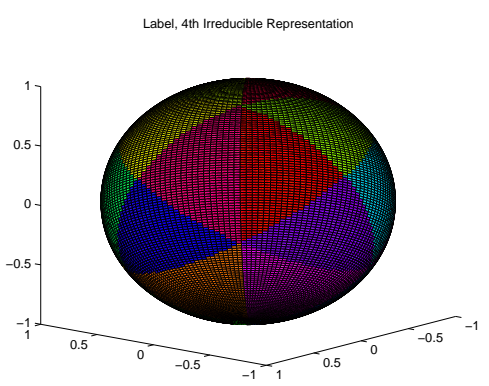

Fig. 1: Tiling of the unit sphere based on the fourth irreducible representation of $\mathrm{O}$

sentation has been constructed by $f_{p}=\boldsymbol{B}^{\prime} f^{p}$ and the two rotations $\boldsymbol{D} \boldsymbol{A} f_{p}$ and $\boldsymbol{D} f_{p}$. The three-dimensional rotation matrices for the two non-trivial rotations are

$$
\boldsymbol{T}_{D}=\left(\begin{array}{ccc}
0 & 0 & 1 \\
1 & 0 & 0 \\
0 & 1 & 0
\end{array}\right) \text { and } \boldsymbol{T}_{D A}=\left(\begin{array}{ccc}
0 & 1 & 0 \\
-1 & 0 & 0 \\
0 & 0 & -1
\end{array}\right)
$$

The three-dimensional filter result vectors are first converted to polar coordinates given by the length of the vector and the unit vector for the direction. Then the following operations normalize the $3 \mathrm{D}$-unit direction vector

1) First apply sequences of $\boldsymbol{T}_{D}$ such that the third component has the highest absolute value. There are three different cases depending on the origin of this component

2) Then there are two different cases, either the component of the highest absolute value was positive or negative. In the case of a negative value of the third component we apply $\boldsymbol{T}_{D A}$ after which we have a positive value in the third component. This gives six different labels and leaves four different cases for the first two components: $(x, y),(-y,-x),(-x,-y),(y, x)$

3) If the absolute value for the first component is larger than the absolute value for the second we exchange the two components. Combined with the previous six classes this results in twelve classes

4) Finally we code the sign of the second component applying the sign change $(x, y) \mapsto(-x,-y)$ if necessary. This gives 24 different labels

The previous labeling process defines a tiling of the unit sphere into the 24 different regions shown in Figure 11. In the computation of the label we change the values in the filter vector correspondingly and we use the last version (after all normalization operations are applied) as a description of the location of the filter vector in a standard region on the sphere. We call this vector the residue vector. Finally we consider the case of the filter system related to the fifth irreducible representation, corresponding to the ordinary rotation representation. After the separation of the three-dimensional filter vector into a length and a direction description we use the following normalization procedure based on the fact that the filter transformations correspond to rotations $A, D$ in Equation (1). The separation of the three-dimensional filterresult vector into magnitude, label and residue is similar to

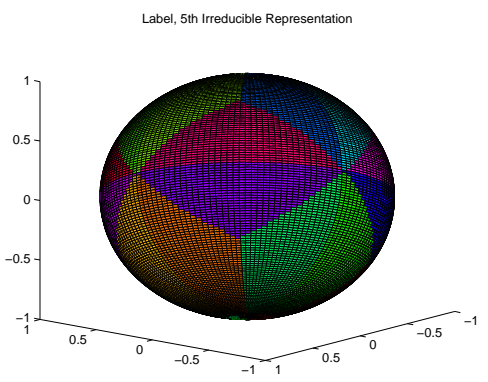

Fig. 2: Tiling of the unit sphere based on the fifth irreducible representation of $\mathrm{O}$

the previous case but now the label is computed as follows:

1) We apply a sequence of operations related to $\boldsymbol{A}$ and $\boldsymbol{D}$ to convert all three component values of the unit direction vector to their absolute values. There are eight different possibilities defining eight different labels

2) Then we apply one of the three shift operations to move the component with the lowest absolute value into the first position in the vector. This splits the eight classes into three subclasses each, resulting in 24 different labels shown in Figure 2

3) The result vector after this normalization is again the residue vector.

The group theoretical transforms were implemented in a package of Maple worksheets and Matlab functions. These functions generate an abstract description of the group from the Maple definition in Section III This description is then translated into a Matlab struct. Other parts of the Matlab toolbox implement the generation of arbitrary representations from two generators, the projection formulas and the generation of invariant subsets from a given sequence of points in 3D.

\section{EXPERIMENTS AND RESULTS}

$\mathbf{I}$ $\mathrm{N}$ our experiments we use two different types of images. Both of them are publicly available and can be downloaded from Internet. The first is the Head MRT Angiography volume on http://www.volvis.org. It is a $3 T$ Magnetic Resonance Tomography (MRT) Time-of-Flight Angiography data set of a human head. It consists of $416 \times 512 \times 112$ voxels with cubic voxels of volume $0.412^{3} \mathrm{~mm}^{3}$. In the following description we refer to this volume as the Head volume.

The second volume is a magnetic resonance angiography (MRA) volume from http://www.physionet.org/physiobank/ database/images. It consists of coronal slices acquired from consecutive anteroposterior positions within the torso of a human body. It's size is $512 \times 512 \times 76$ and the slice thickness is $1.2 \mathrm{~mm}$. We call it the Torso volume. We selected these two volumes since they are publicly available and since they have different statistical properties. We use them for illustration purposes only and we will only discuss them in general statistical terms.

\section{A. Transform coding}

In the first series of experiments we test how good the wide-sense stationary assumption fits these datasets, i.e. how 


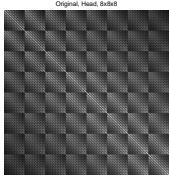

(a) Original data

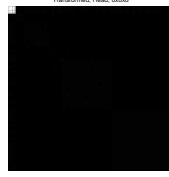

(b) Transformed

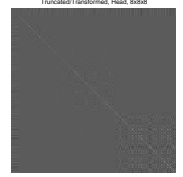

(c) Transformed, without upper left

Fig. 3: Correlation matrices computed from 50000 randomly collected cubes of size $8 \times 8 \times 8$ in the Head volume

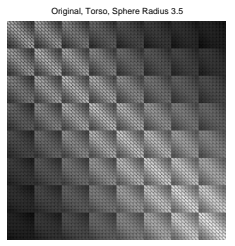

(a) Original data

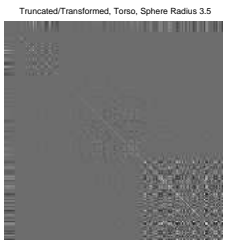

(b) Transformed, without upper left

Fig. 4: Correlation matrices computed from 50000 randomly collected locations in the Torso volume. The domain is a sphere of radius $r=3.5$ The size of the matrix in (a) is $512^{2}$, the matrix in (b) is of size $160^{2}$

much information do we loose if we use the block-diagonal approximation instead of the full correlation matrix.

In Figure 3 a we show the correlation matrix $\boldsymbol{C}$ for a random selection of $500008 \times 8 \times 8$ windows from the Head data set. We applied the coordinate transformation and computed the transformed correlation matrix $\widetilde{\boldsymbol{C}}=\boldsymbol{B} \boldsymbol{C} \boldsymbol{B}^{\prime}$. The decomposition into blocks gives five blocks of sizes 24, 24, 80, 192, 192 . The contributions in the first block are always dominating since they originate in additive combinations of the original data. This is shown in Figure $3 \mathrm{~b}$ We then masked the upper corner of size $24 \times 24$ in $\widetilde{\boldsymbol{C}}$ to enhance the visibility of the remaining entries. This gives Figure $3 \mathrm{c}$. The correlation matrices are of size $512^{2}$ since the window consists of $8^{3}=512$ voxels.

Figure 4 shows correlation matrices for a random selection of 50000 positions in the Torso volume. We select only points in a sphere of radius 3.5 within surrounding $8^{3}$ windows. Figure $4 \mathrm{a}$ is the correlation matrix (of size $512^{2}$ ) computed from the full window. Figure $4 \mathrm{~b}$ shows the same information as Figure $3 \mathrm{c}$ but due to the restriction to the sphere the new, transformed correlation matrices are only of size $160^{2}$. Also here we truncated the dominating first block and we see from Both Figures that, visually, the transformed correlation matrices are indeed block-diagonal to a very good approximation. The goal of the following experiments was to quantify this similarity (and therefore the approximation errors) in more detail.

One variant of Principal Component Analysis (PCA) approximates vectors in the original signal space by linear combinations of eigenvectors of the correlation matrix of the process (the other variation employs eigenvectors of the covariance matrix). In the case of PCA in the original space this means for the full $8^{3}$ windows the computation of a 512 dimensional eigenvector system. If the block-diagonalization is perfectly valid then a description of the vectors in the geometry defined basis $\boldsymbol{B}$ simplifies the 512-dimensional eigenvector problem to a series of five smaller eigenvector problems of sizes $24,24,80,192,192$. In the following figures we illustrate the effects of replacing the full (PCA) system with a system computed from the block-diagonal correlation matrices. We use the two cases mentioned above: full $8^{3}$-windows from the Head volume and spherical domains of radius 3.5 from the Torso volume.

In Figure 11 we show the original image and reconstructed versions for one slice from the Torso volume using the first eigenvector, the first five, nine and ten eigenvectors, only the points inside a sphere of radius 3.5 are used. We illustrate the result using the fourth slice of the eight slices in the middle region of the volume only, but all computations are based on all points in the full $8^{3}$ cube. The results show that the difference between the reconstruction from the full PCA system and the reconstruction from the block-diagonal approximation is very small.

The next experiment investigates the eigenvalue structure for the two volumes. In this experiment we use again the same geometries as in the last experiment: the full cube for the Head and the sphere for the Torso. Both the original correlation matrix and the block-diagonal approximation are used. In Figure 5 we show the normalized accumulated sum of eigenvalues computed from the correlation matrices of the Head volume and the correlation matrices of the Torso volume. The normalization used is $\Lambda_{i}=\sum_{j=1}^{i} \lambda_{j} / \operatorname{trace}(\boldsymbol{C})$ where the $\lambda$ 's are the eigenvalues. We can see that for these two volumes the first four or five eigenvalues explain more than $90 \%$ of the total variance in the data (based on the averaging over 50000 random cube positions).

An interesting property of the eigenvectors is their relation to the blocks in the block-diagonal approximation. The values of the first ten eigenvalues and the block from which they are computed are collected in Table I In this table we normalized the value of the first eigenvalue to one since they had different absolute values due to the difference in the 8- and 16-bit pixel values of the original volumes.

Apart from the dominance of the first eigenvalue and the origin in the first block this table shows several other interesting properties that confirm the validity of the group theoretical symmetry assumption. The most important of these properties is the sequence of the three eigenvalues in second to fourth position. These three eigenvectors all transform in the same way as the fifth irreducible representation which is given by the ordinary rotations in three-dimensional space. Since this is a three-dimensional representation we expect those eigenvectors to come in packages of three which is clearly the case here. We also expect these eigenvectors to have more or less the same eigenvalue which is almost the case, only the third in the class has a lower value than the other two. After these first four eigenvalues the next values in the sequence are already very small and their ordering is probably already influenced by noise contributions. We see however that in both cases, 


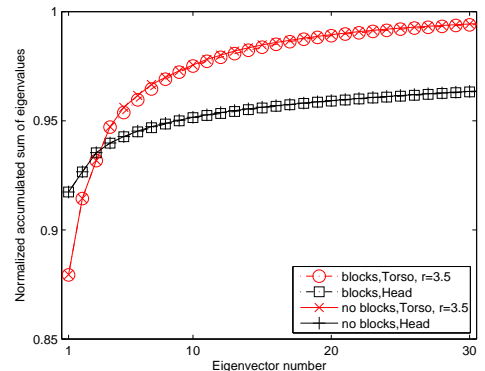

Fig. 5: Distribution of accumulated Eigenvalues for the Head image using all points of the cube and for the Torso images considering the points in a sphere of radius $r=3.5$ centered in the cube

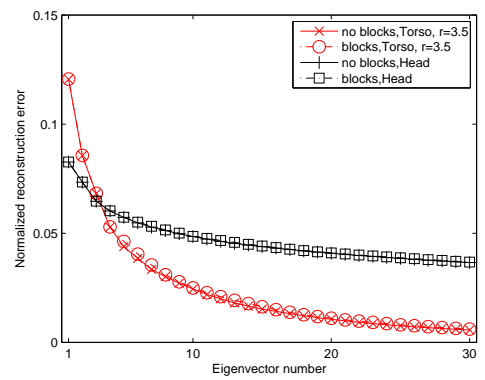

Fig. 6: Mean reconstruction errors for the Head image using all points of the cube and for the Torso images considering the points in a sphere of radius $r=3.5$ centered in the cube

for the Head and the Torso volume the next six eigenvalues come in a package of three eigenvalues related to irreducible representation number four and two eigenvalues related to irreducible representation number three and one to block number one. Also these properties are in accordance with the prediction of the group theoretical framework: eigenvalues in block four should come in triples, those from block three in pairs and those from block one and two are independent of each other. Another interesting property is that eigenvectors from block two are missing. This is an observation we made in other experiments too and shows that they are less prominent in real data sets.

The fact that the basis computed from the full correlation matrix and the basis computed from its block-diagonal approximation have almost identical properties is also confirmed by the distribution of the mean reconstruction error. We used 50000 random samples from the volumes and then we reconstructed the distributions using both bases. In Figure 6 we see that the performance of the approximation system is nearly identical to the reconstruction based on the eigenvectors computed from the full correlation matrix.

\section{B. Filtering}

We now illustrate some results obtained by the filter systems constructed in Section $\mathrm{V}$ above. First we use filters based on some analytical description of the filter form. Then we illustrate how the eigenfunctions computed in the previous section

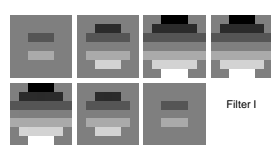

(a) First filter

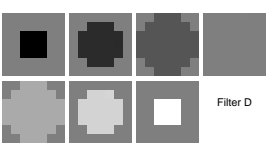

(b) Second filter

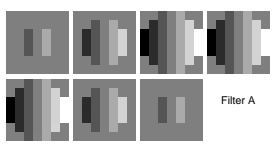

(c) Third filter
Fig. 7: Edge-type Filters, Sphere Radius 3.4, Window Size 7

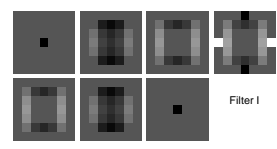

(a) First filter

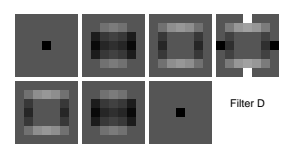

(b) Second filter

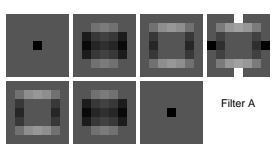

(c) Third filter
Fig. 8: Filters related to the two-dimensional representation, Radii 1.5 and 3, Window Size 7

can be used to construct filter systems that are optimized for group theoretical analysis.

In the first example we consider a cube of size $7 \times 7 \times$ 7 and in this cube all points with a distance less than 3.4 to the origin. This gives a sphere-like region on which these filters are defined. We define an edge detection filter starting from the function $f(\xi, \eta, \zeta)=\xi$. The analysis shows that the filter is completely located in the fifth block and therefore transforms like a three-dimensional representation. After the averaging filters from block one this filter type was dominating in the previous analysis of the eigenvector structure. We show the three filters, given by $f(\xi, \eta, \zeta)=\xi$ and its D- and Atransform in Figure 77 We show them as a sequence of seven slices with varying $\mathrm{z}$-index. The first image in the upper left corner shows thus the distribution of the filter values in the lowest layer of the three-dimensional filter kernel.

In the next example we use $f(\xi, \eta, \zeta)=\eta^{2}-\gamma$. Here $\gamma$ is a constant that assures that filter coefficients sum to zero. The window size is again seven but now we use a hollow sphere with inner radius 1.5 and an outer radius 3 . The three filter functions (given by the quadratic polynomial, defined above, and its D- and A-transform) are shown in Figure 8 . These filters are related to the two-dimensional representation and we see that the third filter, related to the A-transform, is redundant as predicted.

The third filter is given by the product $f(\xi, \eta, \zeta)=$ $P_{3}(\xi) P_{3}(\zeta)$ of a third order polynomial $P_{3}$. The window size was seven as before but now all points in the cube were used, resulting in three filters with 343 coefficients each. The result is shown in Figure 9 . The system consists of three filter functions and its transformation properties are those of the fourth irreducible representation.

In Figure 12 we illustrate the results obtained by applying the edge filter system described above. The system is related to the fifth irreducible representation and corresponds to a conventional edge detection filter defined in a spherical domain. In Figures $12 \mathrm{a}$ to $12 \mathrm{c}$ we see the raw filter results (using Matlabs imagesc conversion) from the three filters. Figure $12 \mathrm{~d}$ is the magnitude image and Figure $12 \mathrm{e}$ shows the color coded labeling. The residual vectors are simply coded in scaled RGB vectors. Since the components in the residual 


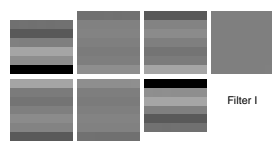

(a) First filter

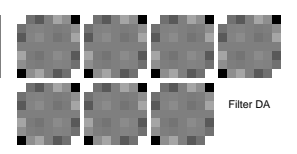

(b) Second filter

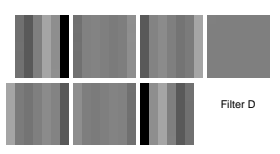

(c) Third filter

Fig. 9: Filters related to second three-dimensional representation, All Points, Window Size 7

vector are ordered we see that the last one (coded in Blue) is dominating.

In the previous illustrations we used an analytical description of the filter functions (or rather one filter function from which we computed the others in the system). In the last illustration we now show how the eigenvectors calculated from the block-diagonal approximation of the correlation matrix can be used for filtering. In this construction we use an eigenvector, in the same way as the analytically described filter function used before. From it we construct the other filters in the system using the group theoretical transformations.

For the Torso image we select from the first ten eigenvectors the vectors number: $1,2,5,6,8$ corresponding to the first eigenvectors belonging to blocks $1,5,3,4$ and 1 . Using the same construction as in the analytical design before we compute from the eigenvector belonging to representation 5 a 3-filter system, for the eigenvector from representation 3 a 2 -filter system, then a 3-filter system again for type 4 . We use the eigenvectors from block 1 as they are. This gives a new filter system consisting of ten filter functions where five of them are selected as eigenvectors and the remaining five are added to create a symmetry-adapted filter system. All of these functions are defined on a spherical region with radius 3.5 inside a cube with $8^{3}$ voxels. The reconstruction properties are almost identical to the results earlier shown in 11 and we therefore choose to illustrate reconstruction properties with the help of the two diagrams in Figure 10. In this figure we show first (Figure 10a) a small region of the center slice shown in 11 and the scan-line used in the following diagrams. The first diagram (Figure 10b) shows the original distribution and the reconstructions using the coefficients from the filter system. The filter system consists ten filters belonging to five blocks and we show the reconstruction based on these blocks. In the second diagram (Figure 10c) we illustrate an example of a symmetry based thresholding strategy. A common strategy in techniques like denoising is to map the original volume into a transform domain, to threshold some of the coefficients and to map the filtered volume back to the original domain. In this example we show how this strategy can be used in conjunction with symmetry based filtering: we first filter the volume with the eigenvector based filter system described before (defined inside a sphere of radius 3.5 in a surrounding cube of size $8^{3}$ voxels). The coefficients are now thresholded in blocks, i.e. all of the coefficients belonging to a given block are either used as they are or set to zero. In this illustration we only use those coefficients where the magnitude of the corresponding block is at least $25 \%$ of the maximum value of this block computed over the whole image. We see that the truncation

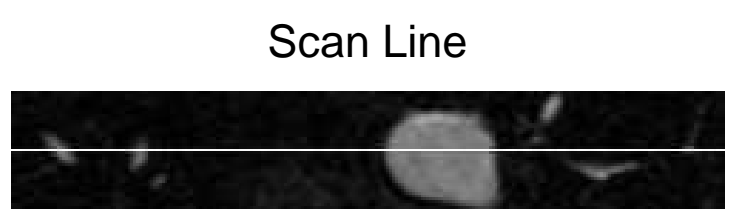

(a) Subregion with scan line

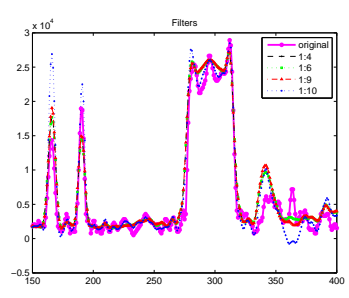

(b) Reconstruction, block-based (c) Reconstruction, truncated blockbased
Fig. 10: Reconstruction with and without truncation of coefficients

results in a smoother reconstruction and we also see that the error of the reconstruction based on the full filter result vector around pixel 360 is avoided in the reconstruction based on the thresholded filter results.

\section{SUMMARY AND CONCLUSIONS}

W E started with the well-known fact that the group of three-dimensional rotations has only a few truly threedimensional finite subgroups. The one that is related to a cubic sampling of space is the octahedral group $\mathrm{O}$. We derived some of its most important properties and sketched a few basic facts from its representation theory. The result is a type of Fourier Analysis for signals defined on domains that are invariant under these octahedral transformations.

We implemented the most important algorithms using a combination of Maple worksheets and Matlab functions and $\mathrm{m}$-files. Using these tools it is easy to construct bases for vector spaces of signals defined on these domains that implement a type of Fourier-transform. We illustrated the usage of the tools by investigating the statistical properties of two MRIvolumes and by designing three-dimensional filter systems. We showed that for these volumes the predictions made by the group theoretical assumptions were essentially valid: we found that the transformed correlation matrices were block-diagonal, that the highest eigenvalue was related to a filter function of 
the averaging type and that the next few eigenvectors came in packages of one, two and three filter functions depending on their origin in the different blocks of the transformed correlation matrices.

\section{REFERENCES}

[1] A. B. Chirikjian, Gregory Sand Kyatkin, Engineering applications of noncommutative harmonic analysis : with emphasis on rotation and motion groups. Boca Raton, FL: CRC Press, 2000.

[2] R. B. Holmes, "Mathematical foundations of signal processing," SIAM Review, vol. 21, no. 3, pp. 361-388, 1979.

[3] _ - "Mathematical foundation of signal processing ii. the role of group theory," Massachusetts Institute Of Technology Lincoln Laboratory, Tech. Rep. Technical Report 781, 1987.

[4] R. Foote, G. Mirchandani, and D. Rockmore, "Two-dimensional wreath product group-based image processing," J. Symb. Comput., vol. 37, no. 2, pp. 187-207, 2004.

[5] D. N. Rockmore and D. M. Healy, Modern signal processing. New York: Cambridge University Press, 2004

[6] R. Lenz, "Investigation of receptive fields using representations of dihedral groups," Journal of Visual Communication and Image Representation, vol. 6, no. 3, pp. 209-227, September 1995.

[7] R. Lenz, T. H. Bui, and K. Takase, "A group theoretical toolbox for color image operators," in Proc. ICIP 05. IEEE, September 2005, pp. III-557-III-560.

[8] S. Sternberg, Group Theory and Physics. Cambridge Univ. Press, 1994.

[9] H. S. M. Coxeter and W. O. J. Moser, Generators and relations for discrete groups. Berlin: Springer-Verlag, 1980.

[10] R. Lenz, "Crystal vision-applications of point groups in computer vision," in Proc. Asian Conference Computer Vision ACCV 2007, ser LNCS, Y. Y. et al., Ed., vol. 4844. Berlin-Heidelberg: Springer, 2007, pp. 744-753.

[11] A. Fässler and E. Stiefel, Group Theoretical Methods and Their Applications. Birkhäuser, 1992.

[12] J. S. Lomont, Applications of Finite Groups. Academic Press, 1959.

[13] A. Levy and J. Rubinstein, "Hilbert-space karhunen-loeve transform with application to image analysis," J Opt Soc Am A, vol. 16, no. 1, pp. 28-35, 1999.

[14] B. Lahme, "Symmetry and the Karhunen-Loeve decomposition," Advances In Imaging And Electron Physics, vol. 132, pp. 69-107, 2004.

[15] R. Lenz, Group Theoretical Methods in Image Processing, ser. LNCS Heidelberg, Berlin, New York: Springer Verlag, 1990, vol. 413. 


\begin{tabular}{|l|l|l|l|l|l|l|l|l|l|l|}
\hline No. & 1 & 2 & 3 & 4 & 5 & 6 & 7 & 8 & 9 & 10 \\
\hline \hline Torso & & & & & & & & & & \\
\hline E-val. & 1 & 0.039 & 0.019 & 0.017 & 0.007 & 0.006 & 0.0057 & 0.0051 & 0.0037 & 0.0032 \\
\hline Block & 1 & 5 & 5 & 5 & 3 & 4 & 4 & 1 & 4 & 3 \\
\hline \hline Head & & & & & & & & & & \\
& & & & & & & & & & \\
\hline E-val. & 1 & 0.010 & 0.009 & 0.004 & 0.003 & 0.002 & 0.0021 & 0.0018 & 0.0016 & 0.0016 \\
\hline Block & 1 & 5 & 5 & 5 & 4 & 1 & 3 & 4 & 3 & 4 \\
\hline
\end{tabular}

TABLE I: Eigenvalues and the block from which they were obtained

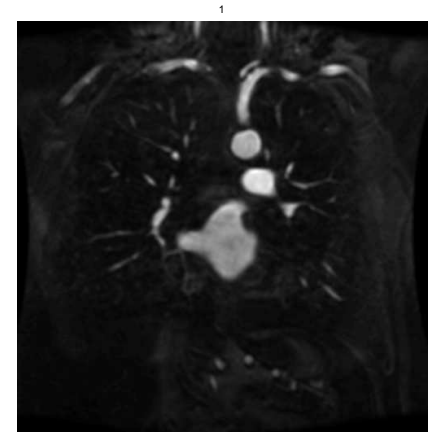

(a)

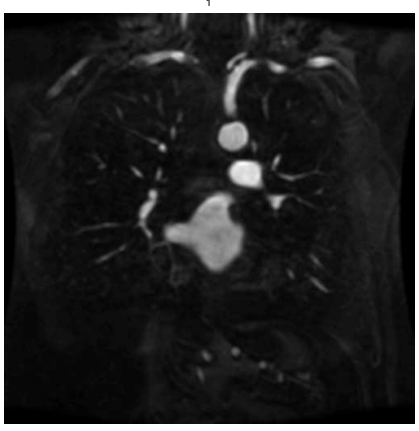

(e)

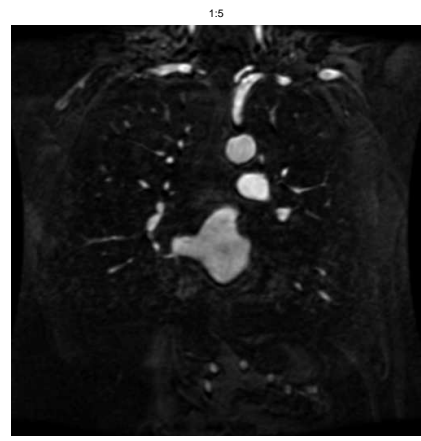

(b)

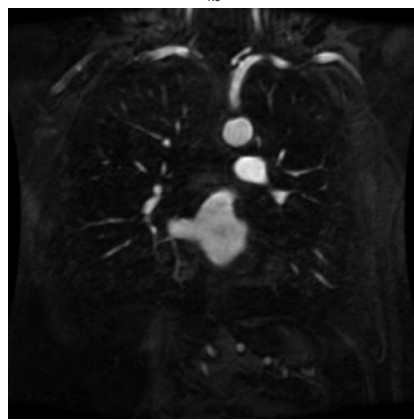

(f)

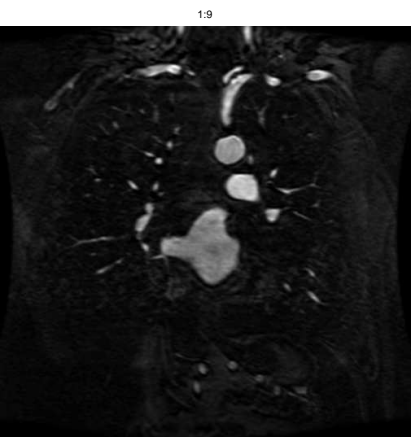

(c)

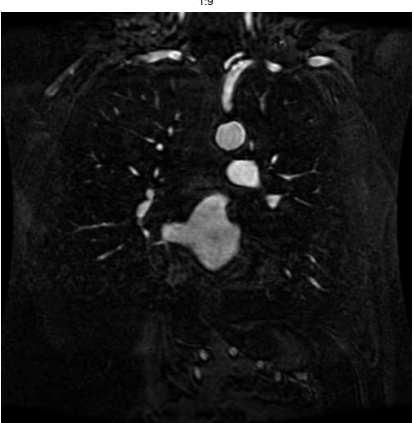

(g)

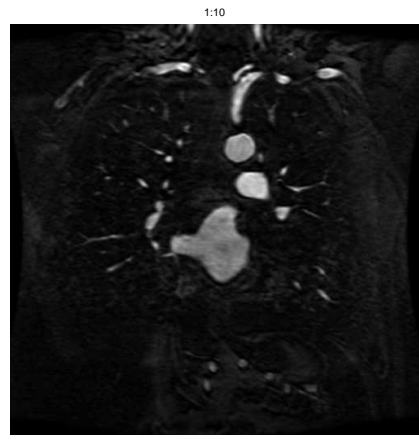

(d)

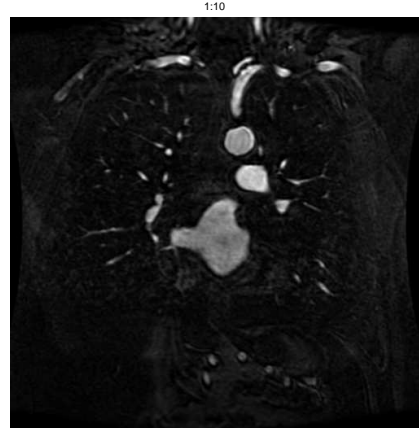

(h)

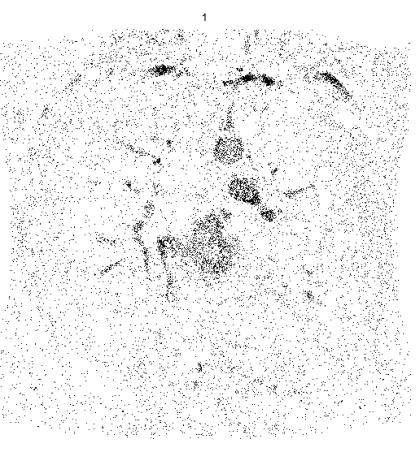

(i)

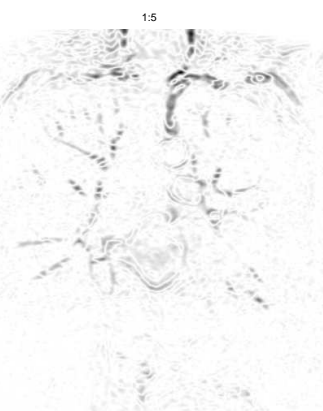

(j) (k) (h)

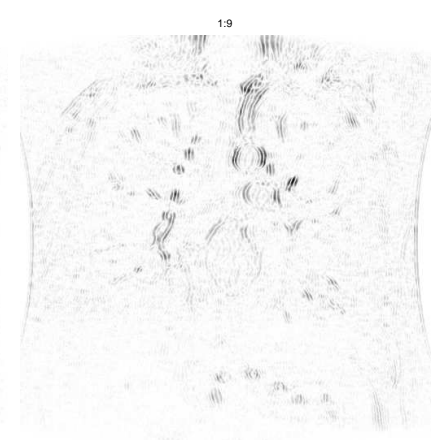

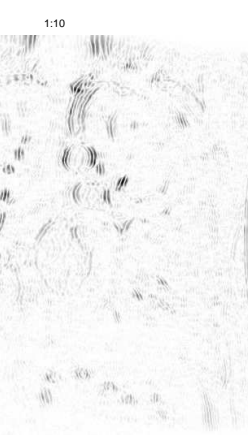

(1)

Fig. 11: Original and reconstructed images for the Torso volume using data slices $30-37$ for 1 to 20 eigenvectors, selecting all points inside a sphere of radius $r=3.5$ with center in the cube of size $W=8^{3}$. First row, eigenvectors from the full correlation matrix. Second row, eigenvectors from the Block correlation matrix. Third row, difference between reconstructions. 


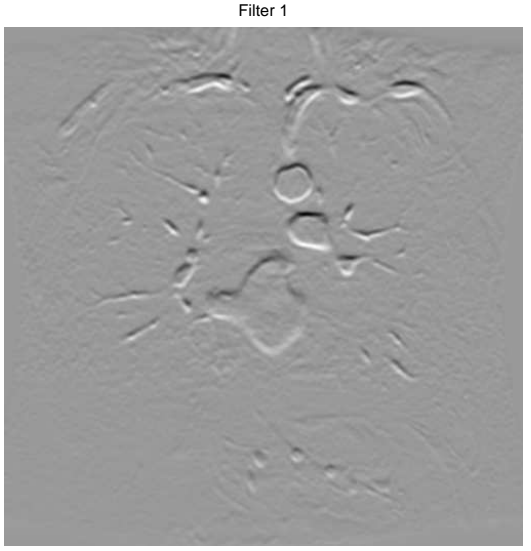

(a) First filter result

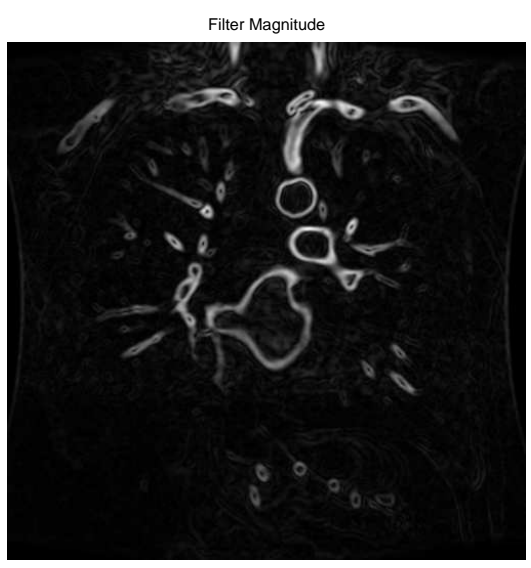

(d) Magnitude of filter results

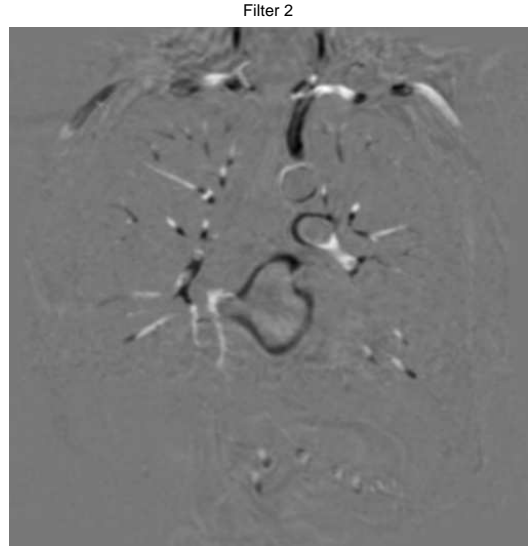

(b) Second filter result

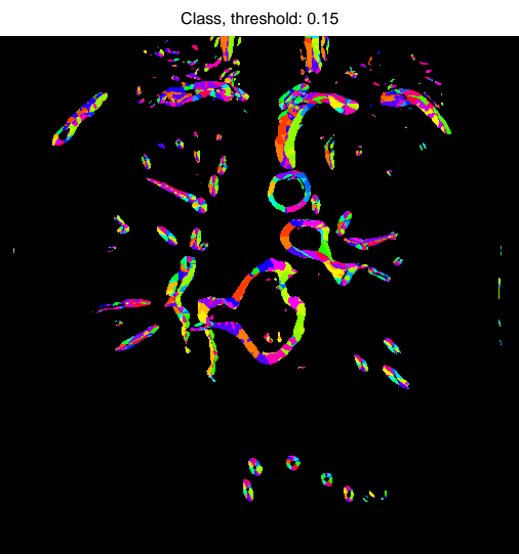

(e) Class label of filter results

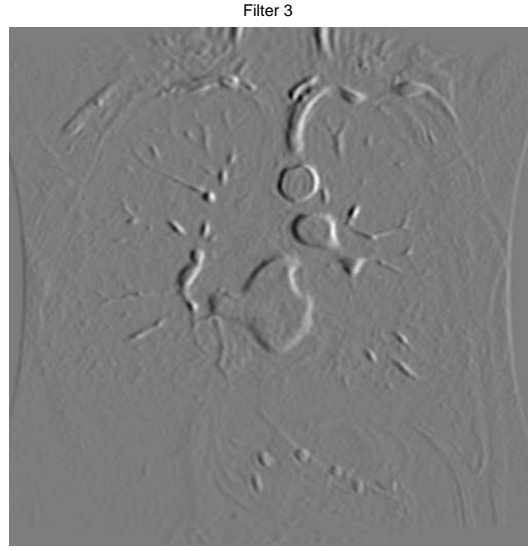

(c) Third filter result

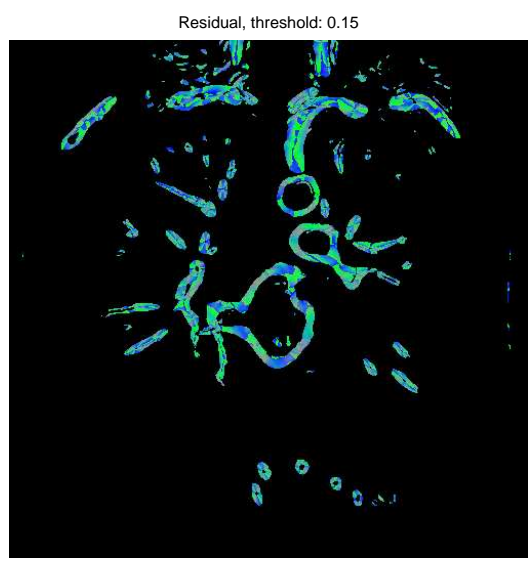

(f) Residue of filter results

Fig. 12: Filter results for filters related to the rotation-representation 